\title{
Whole-Cell Biosensor with Tunable Limit of Detection Enables Low- Cost Agglutination Assays for Medical Diagnostic Applications
}

Nicolas Kylilis, ${ }^{\dagger,+}$ Pinpunya Riangrungroj, ${ }^{\dagger, \S}$ Hung-En Lai, ${ }^{\dagger, \|}$ Valencio Salema, ${ }^{\perp}$ Luis Ángel Fernández, ${ }^{\perp}$ Guy-Bart V. Stan, ${ }^{\dagger, *}$ Paul S. Freemont, ${ }^{*},+, \| \odot$ and Karen M. Polizzi ${ }^{*}, \forall \oplus$

${ }^{\dagger}$ Imperial College Centre for Synthetic Biology, Imperial College London, London SW7 2AZ, United Kingdom

${ }^{*}$ Department of Bioengineering, Imperial College London, London SW7 2AZ, United Kingdom

${ }^{\S}$ Department of Life Sciences, Imperial College London, London SW7 2AZ, United Kingdom

"Section of Structural Biology, Department of Medicine, Imperial College London, London SW7 2AZ, United Kingdom

${ }^{\perp}$ Department of Microbial Biotechnology, Centro Nacional de Biotecnología (CNB-CSIC), Darwin 3, Campus Cantoblanco UAM, 28049 Madrid, Spain

\# Department of Chemical Engineering, Imperial College London, London SW7 2AZ, United Kingdom

Supporting Information

ABSTRACT: Whole-cell biosensors can form the basis of affordable, easy-to-use diagnostic tests that can be readily deployed for point-of-care (POC) testing, but to date the detection of analytes such as proteins that cannot easily diffuse across the cell membrane has been challenging. Here we developed a novel biosensing platform based on cell agglutination using an E. coli whole-cell biosensor surface-displaying nanobodies which bind selectively to a target protein analyte. As a proof-ofconcept, we show the feasibility of this design to detect a model analyte at nanomolar concentrations. Moreover, we show that the design architecture is flexible by building assays optimized to detect a range of model analyte concentrations using straightforward design rules and a mathematical model. Finally, we reengineer our whole-cell biosensor for the detection of a medically relevant biomarker by the display of two different nanobodies against human fibrinogen and demonstrate a detection limit as low as $10 \mathrm{pM}$ in diluted human plasma. Overall, we demonstrate that our agglutination technology fulfills the requirement of POC testing by combining low-cost nanobody production, customizable detection range and low detection limits. This technology has the potential to produce affordable diagnostics for field-testing in the developing world, emergency or disaster relief sites, as well as routine medical testing and personalized medicine.

KEYWORDS: whole-cell biosensor, synthetic biology, point-of-care testing, medical diagnostics, latex agglutination test, immunoassay

A ffordable point-of-testing diagnostic technology applied to resource-limited sites is one of the most promising biotechnologies for improving global health. ${ }^{1}$ The development of new diagnostics and their successful adoption in the field by end users requires consideration of various diverse factors including, but not limited to, scientific challenges, economic restrictions and practical considerations. ${ }^{2}$ High impact technology will allow sensitive and specific detection, it will be low-cost and portable to ensure accessibility, and it will have a user-friendly design that does not rely on sophisticated equipment. ${ }^{3}$

Immunoassays are a dominant technology in in vitro diagnostics. ${ }^{4}$ Antibodies can be generated for the target analyte with very high specificity and relative ease. Among the various types of immunoassay formats for the development of rapid diagnostic tests, latex agglutination tests (LAT), which use antibody molecules immobilized on latex particles to detect the presence of an analyte, are available for more than 300 diseases and biomolecules. ${ }^{5}$ Multivalent immuno-latex particles can recognize their target analyte molecules through specific interactions and form higher-order complexes. As the agglutination reaction proceeds, extensive cross-linking between analyte molecules and latex particles leads to the formation of very large complexes that can be visibly detected by the naked eye or monitored spectrophotometrically. ${ }^{6}$

The use of whole cells as a bioanalytical platform for in vitro medical diagnostics offers a number of favorable characteristics such as low cost of production. Whole cells are self-replicating and can manufacture recognition elements such as antibodies,

Received: October 3, 2018

Accepted: January 9, 2019

Published: January 9, 2019 
eliminating the need for expensive purification steps. In addition, as living organisms, whole cells can provide physiologically relevant data on the bioavailability of the analyte. Bacteria, especially model organisms such as Escherichia coli, are often used as bioreporters as they are amenable to genetic engineering and easy to culture. Bacterial whole-cell biosensors have been genetically engineered to detect medically relevant analytes such as metabolites, ${ }^{8}$ toxic chemicals ${ }^{9}$ and mercury in urine, ${ }^{10}$ hydroxylated polychlorinated biphenyls in serum ${ }^{11}$ and nitrogen oxides in both serum and urine samples. ${ }^{12}$ Whole-cell biosensors usually rely on intracellular detection of the target analyte, ${ }^{13}$ but such designs are limited to the detection of analytes that can diffuse or be actively transported across the bacterial cell membrane. Nevertheless, there is a multitude of medically relevant analytes such as protein biomarkers of disease states ${ }^{14-17}$ that cannot cross the membrane barrier. A few bacterial wholecell bioreporter designs have been demonstrated to detect analytes extracellularly, ${ }^{18,19}$ but these are of limited versatility in terms of choice of target because of their mechanism of action.

Merging bacterial surface-display technology and antibody engineering can provide a mechanism for the detection of extracellular analytes with whole-cell biosensors in a biological equivalent of the latex agglutination test (LAT). Single chain antibody fragments $(\mathrm{scFv})$ have been displayed on the surface of bacteria using engineered surface-display proteins that localize to the outer membrane of the cell. ${ }^{20-22}$ However, scFvs often have poor solubility, misfold and aggregate when expressed in bacterial hosts. As an alternative, single-domain antibodies or nanobodies, are antibody fragments from heavy chain-only antibodies produced by the Camelidae family. They have high stability when expressed in bacterial hosts ${ }^{23,24}$ and have been demonstrated to functionally display on the outer membrane of E. coli $^{25-27}$ and other bacterial species. ${ }^{28,29}$

Here we demonstrate the development of a biological equivalent of the LAT that utilizes the E. coli surface-display of camelid nanobodies as a detection element. As a starting point, we used a nanobody against green fluorescent protein (GFP) with a tandem dimeric GFP (tdGFP) analyte to induce crosslinking as a proof-of-concept. Using this convenient platform and a mathematical model of the agglutination process, we explored the effects of variables such as the number of bacterial particles in the assay and the nanobody expression level on the limit of detection of the assay. The total number of nanobodies in the reaction was found to be the primary factor influencing the limit of detection. With this understanding, we then explored the modularity of the platform by creating a wholecell biosensor for the detection of human fibrinogen (hFib), a clinically relevant biomarker high levels of which are associated with cardiovascular disease and inflammation ${ }^{30}$ and low levels of which can be used in the diagnosis of disseminated blood clotting. ${ }^{31}$ Here, we propose a modular biosensing platform that harnesses the versatility and sensitivity of the LAT, but without the requirement for expensive steps to isolate and purify antibodies.

\section{RESULTS AND DISCUSSION}

Proof-of-Concept for a New Diagnostic Assay System. For the detection of protein analytes, an assay format was adopted that resembles the format of the LAT. The assay design utilizes bacterial cells displaying a nanobody on their cell surface to facilitate analyte recognition in the extracellular environment (Figure 1A). The display of several copies of the nanobody results in an engineered bacterial cell multivalent for

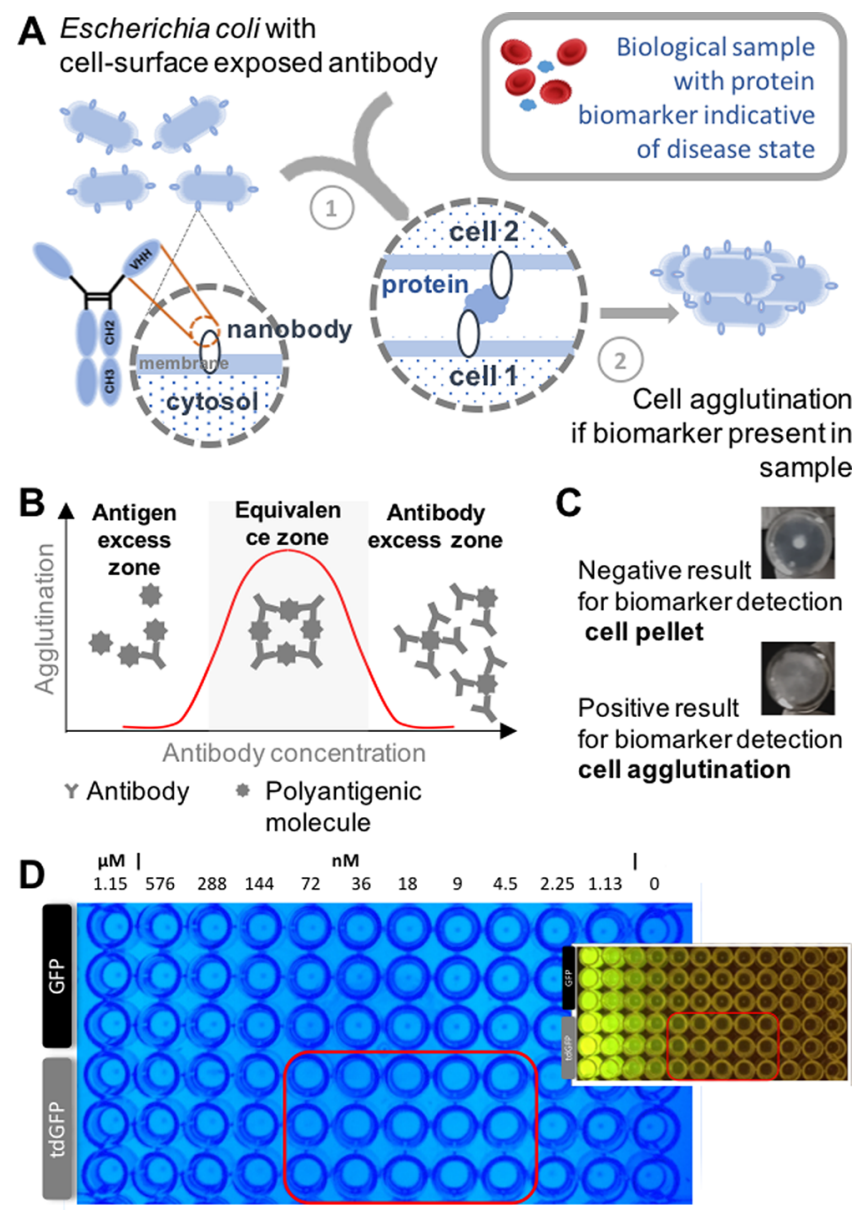

Figure 1. Low-cost, protein biomarker test for point-of-care medical diagnostics. (A) Assay design and test workflow for point-of-care testing. E. coli cells with surface-displayed nanobodies are mixed with a biological sample (e.g., blood) from a patient. If the patient sample includes the protein biomarker indicative of disease state, this will cause cross-linking between bacterial cells and biomarker molecules that leads to cell agglutination and changes the visual appearance of the test sample (test result). (B) The Heidelberger-Kendall curve describes the extent of aggregation of molecules in an agglutination reaction as a function of the antigen/antibodies ratio. (C) Positive and negative test results for cell agglutination assay. (D) Images of the proof-of-concept experiment, where E. coli cells displaying the antiGFP nanobody on their cell surface have been incubated with monomeric GFP (negative control) or tdGFP (model biomarker) in microplates. The red box highlights wells with tdGFP concentrations that demonstrate high-levels of cell agglutination. In the main image, the microplate was back-illuminated with visible blue light for better contrast. In the secondary image, the same microplate was illuminated with the application of a GFP filter to demonstrate protein (GFP or tdGFP) concentrations.

its target analyte. In the presence of di- or multivalent analyte molecules or when bacterial cells expressing nanobodies against multiple epitopes of a monovalent antigen are mixed, cross-linking between cells and analyte molecules produces aggregated bacterial clumps. This agglutination of bacterial cells results from the specific binding of protein analyte molecules to displayed nanobody elements in a bacteriumprotein-bacterium sandwich. It was expected that the behavior 
A

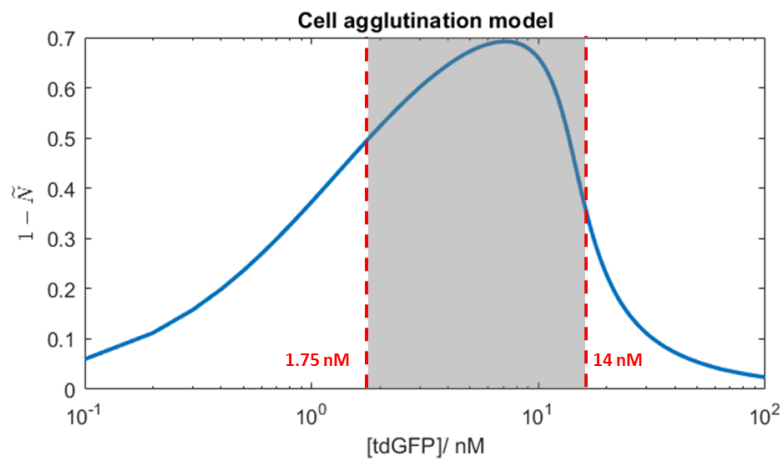

$\begin{array}{lllllllllllll}n M & 113 & 57 & 28 & 14 & 7 & 3.5 & 1.750 .88 & 0.440 .220 .11 & 0\end{array}$ 000000000000
B

Difference in $[$ tdGFP] $\max$

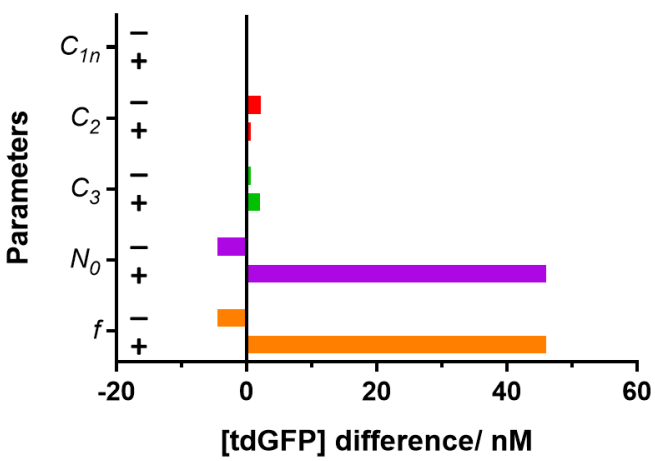

C

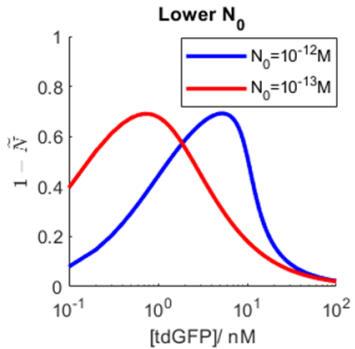

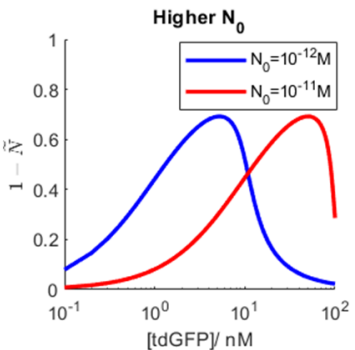
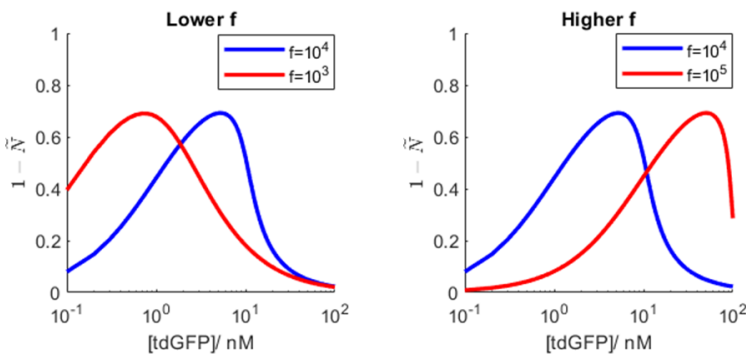

Figure 2. Mathematical modeling and sensitivity analysis of the bacterial agglutination reaction (A) Computational simulation of the bacterial agglutination reaction for tdGFP analyte concentrations. $Y$-axis represents the end point normalized cell agglutination value $(1-\tilde{N})$ for each analyte concentration when the system is solved numerically over a time span of $18 \mathrm{~h}$. The red dotted lines indicate the maximal and minimal tdGFP concentrations (1.75-14 nM) where cell agglutination was observed in an experimental assay (image of the plate assay at the bottom of the graph, where agglutination-positive samples are indicated with a red rectangle). (B) Bar chart of the change in the concentration of tdGFP analyte at which maximum normalized cell agglutination is observed after parameter perturbation from their nominal values to 10 -fold higher $(+)$ or lower $(-)$. The parameters are (from top to bottom): normalized agglutination rate constant $\left(C_{1 n}\right)$, nanobody binding rate constant $\left(C_{2}\right)$, nanobody dissociation rate constant $\left(C_{3}\right)$, initial concentration of cells $\left(N_{0}\right)$, and number of nanobodies per cell $(f)$. (C) Simulations of the agglutination reaction at either 10-fold-lower or 10-fold-higher concentrations of bacterial cells $\left(N_{0}\right)$ or nanobody per cell $(f)$ (red curve) with respect to the base model (blue curve).

of this biological LAT would resemble the general curve exhibited in agglutination immunoassays (Figure 1B) where maximum agglutination of bacterial cells would be observed at a specific ratio of cells to antigenic molecules, with deviation from this ratio leading to progressively lower levels of agglutination. The visual output of a lack (or low levels) of bacterial agglutination is a dense bacterial pellet, while high levels of agglutination result in a network or membrane-like structure that spreads across the bottom of a well when the reaction is carried out in round-bottom microplate well (Figure 1C).

For proof-of-concept experiments, E. coli cells were designed to express anti-GFP nanobody that is surface-displayed through a $\beta$-intimin anchor. ${ }^{27}$ Surface localization and functional display of the anti-GFP nanobody was demonstrated by live cell fluorescence labeling and flow cytometry (Supplementary Note 1 and Supplementary Figure 1A-B) and the number of nanobodies displayed per cell was estimated by GFP titration followed by flow cytometry analysis (Supplementary Note 1 and Supplementary Figure 1C). To induce cross-linking of cells, a divalent analyte, tdGFP, was engineered by fusing a linker domain of 12 amino acids to two GFP monomers (Supplementary Note 2). Each domain of tdGFP can bind to an individual nanobody on separate cells initiating cell agglutination. To test this, we overexpressed and purified recombinant tdGFP (Supplementary Figure 2A). Fluorescence assays suggested that both GFP domains were correctly folded as the fluorescence intensity is approximately twice that of the GFP monomer at the same molarity (Supplementary Figure 2B).

To set up the agglutination assay, suspensions of E. coli cells expressing the anti-GFP nanobody were titrated with tdGFP analyte in concentrations ranging from $1.15 \mu \mathrm{M}$ to $1.13 \mathrm{nM}$ in 2 -fold dilutions in round-bottom 96-well microplates. This experimental setup is typical of diagnostic hemagglutination assays in clinical settings. After overnight incubation, cell agglutination was clearly visible in the analyte range of 4.5-75 $\mathrm{nM}$ tdGFP, in the form of a membrane-like structure across the bottom of the wells (Figure 1D). At lower (prezone) and higher (postzone) tdGFP concentrations, distinct cell pellets were formed at the bottom of the wells (Figure 1D) indicating a lack of cell cross-linking. An analogous control reaction where cells were titrated with the equivalent concentrations of monomeric GFP, which should not initiate cell agglutination, showed cell pellet formation across the entire GFP analyte concentration range tested, indicating that cell agglutination is specific for the tdGFP analyte.

Modulating the Dynamic Range of the Diagnostic Assay. The use of bacterial agglutination assay as a new diagnostic technology would benefit from designs that allow versatility in the detection of disease biomarkers. While the design of the demonstrated prototype assay platform is inherently modular since the nanobody can be engineered to bind specifically to any target, different medical conditions may 
require detection of molecular targets at different physiological concentrations in biological samples. ${ }^{14-17,32}$

To enable further understanding of our nanobody-induced cell agglutination system and allow for the development of tunable assays for specific medical applications, we adapted a previously described mathematical model of the agglutination process $^{33}$ to the specifics of our technology. Briefly, our mathematical model describes the dynamics of cell agglutination in the presence of freely diffusing analytes in which a finite number of cells are cross-linked via the tdGFP analyte under certain conditions, and consequently form larger aggregates over time. The output of the model is a measure of the expected level of bacterial cell agglutination at the end of the reaction compared to the initial number of free bacterial cells in the reaction, which we refer to as the normalized cell agglutination value $(\tilde{N})$. The model output is dependent on several variables which can be directly controlled in the laboratory such as the antibody binding rate constant $\left(C_{2}\right)$ and the dissociation rate constant $\left(C_{3}\right)$, the added concentration of analyte $\left(N_{1}\right)$, the number of nanobodies displayed per cell $(f)$, and the concentration of cells in the reaction $\left(N_{0}\right)$. A more detailed explanation and derivation of the model, including a list of assumptions, can be found in Supplementary Note 3.

Initially, we tested the predictive capabilities of the model by simulating the cell agglutination reaction using variable values derived experimentally or from the literature (Supplementary Table 1). The simulation predicted that the maximum value of normalized cell agglutination would occur at $7 \mathrm{nM}$ tdGFP analyte (Figure $2 \mathrm{~A}$, region between red dashed lines), in good agreement with the experimentally observed agglutination zone (Figure 2A, red rectangle). These results demonstrated that the model successfully captures the agglutination reaction dynamics. Next, we applied sensitivity analysis to the model to identify parameters with the largest influence on the agglutination reaction and the normalized cell agglutination value. The parameters that showed the greatest relative sensitivity were the normalized agglutination rate constant, $C_{1 n}$, and the total number of nanobodies in the reaction, $N_{0} f$, (Supplementary Figure 4). We decided to further explore the effects of the latter, as this variable is readily tunable experimentally through changing the number of cells in the reaction or through modulating the expression levels of the nanobody. An analysis that investigated the change in the analyte concentration at which maximum agglutination levels are observed, demonstrated that perturbing these two parameters individually by 10 -fold with respect to their nominal values results in the biggest change of all parameter perturbations possible (Figure 2B). In practice, this means that these variables can be used to alter the concentration range of the zone of agglutination. Figure $2 \mathrm{C}$ shows the results of these simulations for both variables in their 10 -fold perturbation scenarios.

The total number of nanobodies in the agglutination reaction can be manipulated experimentally by either changing the number of cells in the assay or the number of nanobodies displayed on the surface of each cell. Although changing the number of cells in the reaction is the easier of the two approaches, in practice this is hindered by a lower limit on the concentration of cells that can be detected visually (OD600 $\mathrm{nm} \sim 0.25$, Supplementary Figure 3). While adjusting the number of nanobodies per cell is more technically challenging, to facilitate the use of this technology in an easy plug-and-play manner, we developed a library of vectors that allows for different numbers of nanobody displayed on the surface of each cell. For this library, we used three promoters of low, medium and high strengths from the Anderson collection of constitutive promoters, ${ }^{34}$ and three ribosome binding site (RBS) designs of low, medium and high strengths designed using the RBS calculator, ${ }^{35}$ leading to nine device variants with different promoter-RBS combinations that were expected to result in different levels of displayed anti-GFP nanobodies.

Initially, we used flow cytometry to measure the levels of nanobody displayed per cell using live cell labeling with monomeric GFP. The data showed a large variation in mean cell fluorescence among device variants (Figure 3, bar charts), indicating varied expression of anti-GFP nanobodies on the surface of the cells. Among the variants, device v02 showed the lowest levels of nanobody display and device v07 showed the
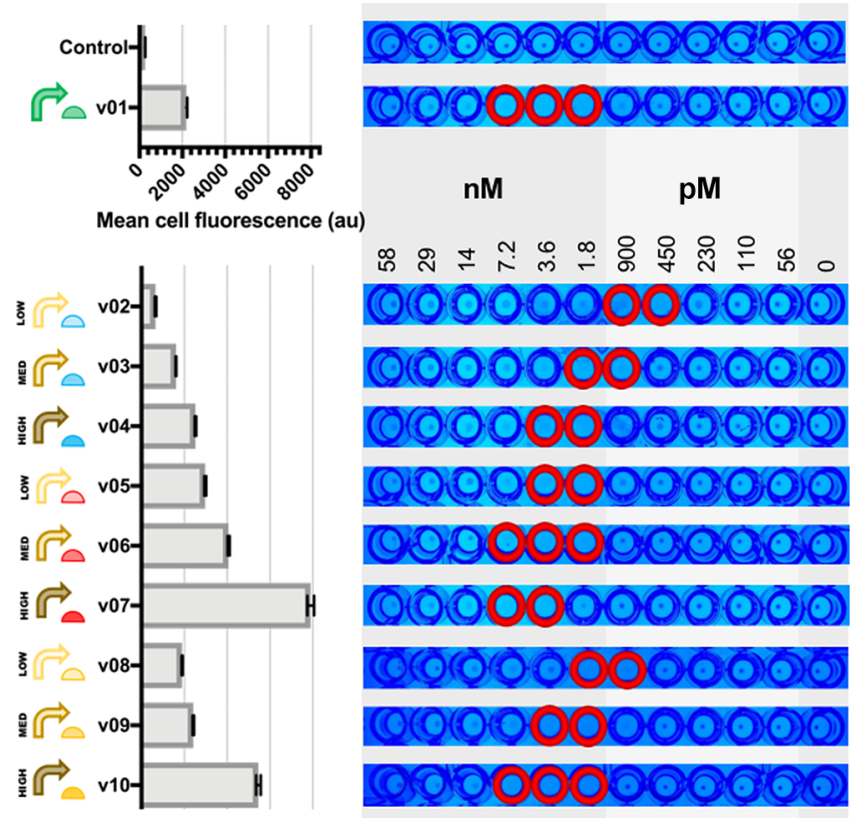

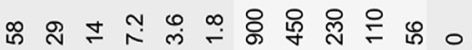
C(00000000000) Q00000000000) 000000000000 Q0000000000 00.000000 .030 000000000000 (c)(000000000) Q(00100000000)

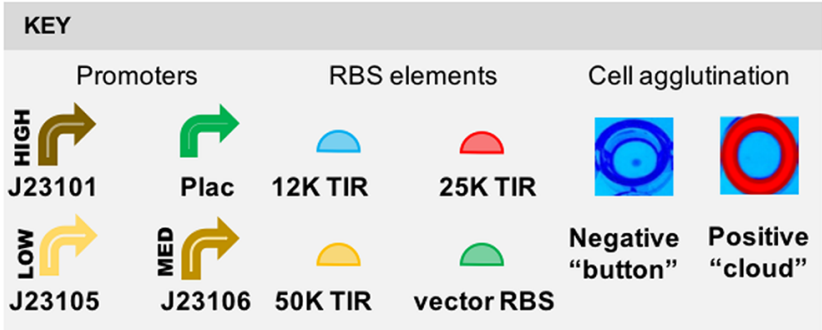

Figure 3. Modulation of the analytical characteristics of the agglutination assay by the control of the nanobody expression levels. The prototype (v01) E. coli cells, which have been engineered to display an anti-GFP nanobody on their surface, were genetically modified to produce variants (v02-v10) that express different levels of anti-GFP nanobody. Genetic elements used to control the expression levels of the nanobody display vector are shown next to each variant. The bar chart shows the mean cell fluorescence of each cell variant population when labeled with saturating amounts of purified GFP. Cell fluorescence was recorded by flow cytometry, and error bars show standard deviation for three technical replicates. Images of cell agglutination assay in a microplate, with engineered $E$. coli cells displaying anti-GFP nanobody on their surface after incubation with various tdGFP concentrations. The microplate was back-illuminated with blue light. Control cells did not express any nanobody. 

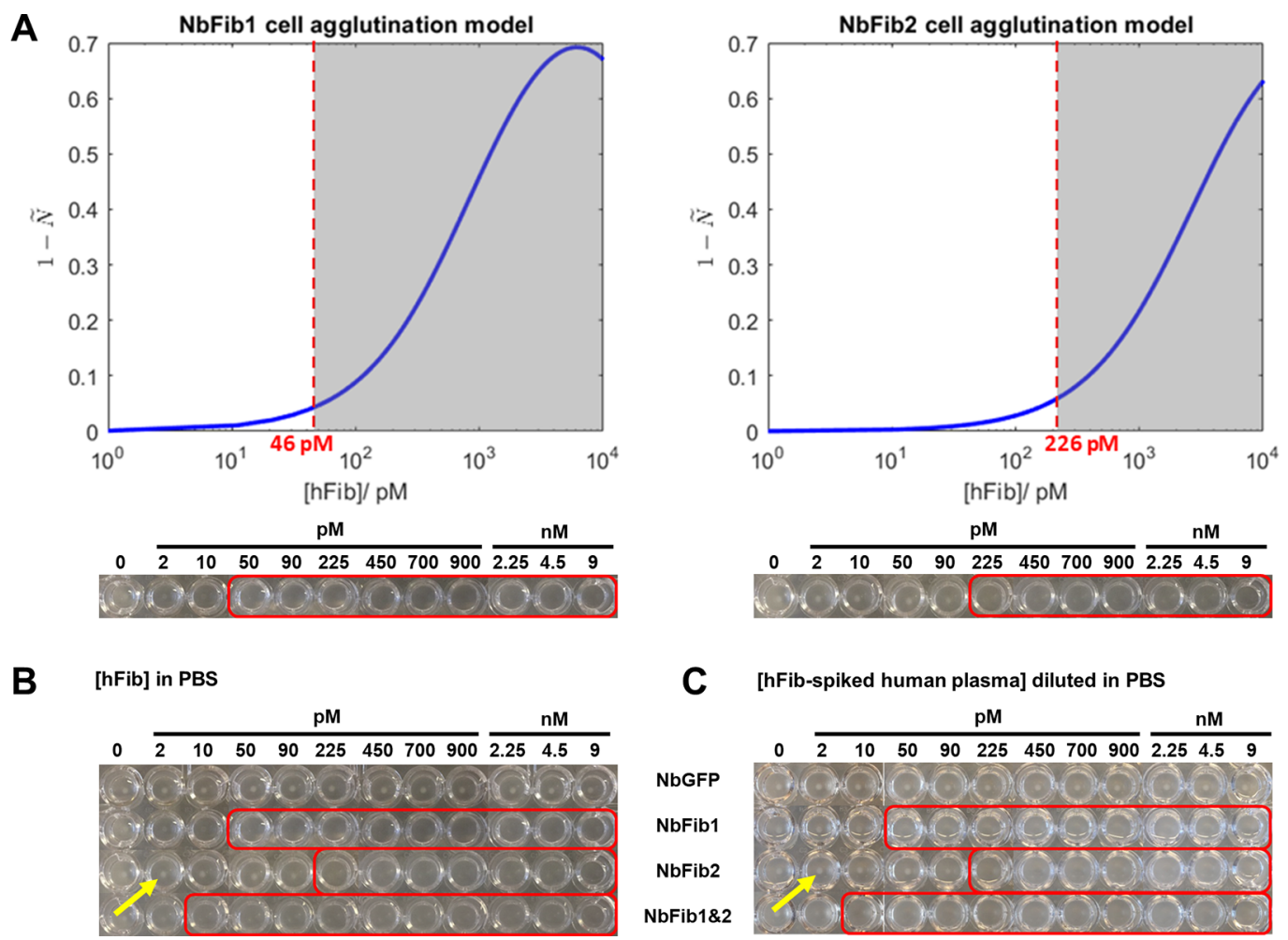

Figure 4. Validating the agglutination assay using human fibrinogen (hFib). (A) Computational simulations of the bacterial agglutination reaction for fibrinogen detection for two different antifibrinogen biosensor cell strains. Dark region indicates the zone of agglutination and red dotted line indicate the minimal fibrinogen concentration where cell agglutination was observed in experimental assays (image of the plate assay at the bottom of the graphs, where agglutination positive samples are indicated with a red rectangle). (B) Plate agglutination assay of E. coli cells expressing antihFib nanobodies (NbFib1/2). Plate wells included single lines of biosensor cells (plate rows $1-3$ or mixed populations of cell lines (row 4 ) incubated at various hFib concentrations (right). (C) Bacterial agglutination assay of human plasma samples spiked with hFib analyte concentrations. Cells expressing anti-GFP nanobody were used as a negative control. Red boxes indicate zones of bacterial agglutination. Yellow arrows indicate cell pellet formation.

highest levels of nanobody display with the rest of the variants showing levels between these extremes. The subsequent use of different variants in the plate agglutination assays with the tdGFP analyte showed shifts in the zone of agglutination that strongly correlate with the nanobody expression levels, confirming the predictions of the simulations using the mathematical model (Figure 3, microplate images). In particular, the dynamic range of device v02 shifted toward lower analyte concentrations allowing the detection of tdGFP analyte at concentrations as low as $450 \mathrm{pM}$, compared to the previous detection limit of $1.8 \mathrm{nM}$ in the initial prototype.

Bioassay Development toward Use as a Real-World Diagnostic Test. Human fibrinogen (hFib) is a glycoprotein with normal blood plasma levels between 1.5 and $4.5 \mathrm{~g} / \mathrm{L}^{36}$ $\mathrm{hFib}$ plays a role in blood coagulation when it is converted into fibrin by thrombin protease. ${ }^{31}$ Elevated levels of fibrinogen in plasma samples are commonly used as a biomarker for determining the risk of cardiovascular disorders in humans, ${ }^{30}$ whereas low levels of fibrinogen in plasma are associated with blood disorders such as disseminated clotting. ${ }^{31} \mathrm{hFib}$ is a heterohexamer, consisting of two dimers of three different polypeptide chains and therefore it is inherently a multivalent antigen. Therefore, we decided to develop a diagnostic test for hFib as a first real-world use case of our new bacterial LAT platform (see Supplementary Note 4 for further explanation as to how different disease states can be distinguished).

We began by constructing two cell lines that surface-display two different anti-hFib nanobody constructs (NbFibl and
NbFib2) with different epitope recognition sites. ${ }^{25}$ Each nanobody was expressed on the cell surface using the same display vector, and anti-hFib nanobody display was confirmed by flow cytometry and fluorescent microscopy analyses (Supplementary Figure 6). Additionally, we used our mathematical model with updated parameter values specific to the hFib system to predict the range of analyte concentrations at which the agglutination zone would be observed (Figure 4A).

We then verified the designed diagnostic test with purified samples of hFib protein in a microplate agglutination assay format. For each of the two cell lines with nanobodies against different hFib epitopes, the assay produced bacterial agglutination zones at the analyte concentration ranges predicted by the model. The equivalence zone for the hFib assay was broader than that of the GFP assay, likely due to aggregation of the fibrinogen protein in solution (Supplementary Figure 7). The detection limit was the lowest for the cell line displaying the NbFib1 nanobody at $\sim 50 \mathrm{pM} \mathrm{hFib} \mathrm{in}$ the reaction; the detection limit for the assay using the NbFib2 nanobody was $\sim 230 \mathrm{pM}$ (Figure 4B). We speculate that these shifts can be attributed to differences in nanobody expression (Supplementary Figure 6). Importantly, a control reaction using cells that display anti-GFP nanobodies did not exhibit any agglutination, providing further evidence that specific interactions between analyte and nanobody elements drive the cross-linking effect. 
The availability of nanobodies that bind to distinct epitopes in hFib provided the opportunity to test whether the limit of detection could be further decreased by increasing the propensity for cross-linking through the availability of multiple binding sites (Figure 4B). Indeed, a mixture of the cells expressing $\mathrm{NbFib} 1$ and $\mathrm{NbFib} 2$ resulted in a further decrease in the limit of detection to $10 \mathrm{pM} \mathrm{hFib.}$

Finally, to test the robustness of the assay to potential sample matrix effects, we compared the assay results using samples of hFib reconstituted in fibrinogen-deficient human plasma with those reconstituted in phosphate-buffered saline (PBS). As shown in Figure 4C, there was no observable difference between samples prepared in PBS or human plasma samples, suggesting that the assay is robust to the sample background and can be used with real patient samples without interference.

\section{CONCLUSIONS}

In this paper, we describe the development of a biological equivalent to the LAT, an assay widely used in medical diagnostics. Our bioassay includes cells that are genetically engineered to display nanobodies on their surface, analogous to the latex particles coated with antibodies in LATs. The presence of the analyte induces bacterial cross-linking, which is visible to the naked eye and enables facile detection of the target analyte in a manner similar to the readout of the LAT. This expands the repertoire of the types of analytes that can be sensed using whole-cell biosensors to include large molecules such as protein biomarkers or disease agents, which cannot easily be transported into the cell.

Whole-cell biosensors have become an alternative promising tool for the detection of analytes in diverse applications, as they are easy to manipulate, simple and low-cost. Our platform adds a new tool to this arsenal for the extracellular detection of protein analytes. Additionally, our platform is unique in enabling analyte detection without requiring active transcription and translation of a reporter protein. This contrasts with most previous solutions where the output signal is dependent on active gene expression for reporter protein production. ${ }^{12,37,38}$ Lastly, the platform can potentially offer large cost savings to assay manufacturers as the cells autonomously express and surface display nanobodies which removes the need for the costly steps of antibody production, purification, and attachment to latex particle surfaces.

The design of our biological LAT is inherently modular and is readily extensible to the detection of other analytes of interest by exchanging the nanobody that is surfacedisplayed through simple molecular cloning techniques. There are numerous developed nanobodies described in the literature. ${ }^{39}$ Alternatively, phage-display libraries can be used to select new nanobodies for different targets or to enhance specificity, ${ }^{40,41}$ or immunization, e.g., of camels orllamas can be used to isolate new nanobodies if desired. ${ }^{42}$ Thus, the technology described here could be used as a platform for the development of a range of assays for different target analytes. For inherently multivalent targets, a nanobody that binds to a single epitope is all that is necessary to develop a new assay. Monomeric targets can be detected by mixing two cell populations expressing nanobodies that bind to different epitopes. Additionally, we have shown that the dynamic range of this diagnostic platform can be easily tuned for the requirement of the particular application, and very low limits of detection are easily achievable. In particular, the limit of detection of our biological LAT assay for hFib was about 20,000-fold lower than that of the previously developed LAT assay, ${ }^{43}$ and was also over 30,000-fold and 15-fold lower than those of other types of biosensors such as a piezoelectric agglutination sensor ${ }^{44}$ and an amperometric immunosensor, ${ }^{45}$ respectively. Using our mathematical model in combination with the developed library of variants of nanobody expression, new diagnostic tests can be conveniently designed for target analyte concentrations at will.

An important consideration for the adoption of new technologies is ease-of-use and seamless integration into existing professional workflows. Our biological LAT platform is underscored by the simplicity of its design and gives a visual readout that is easy to interpret. In addition, it potentially fits within existing clinical diagnostic frameworks since the LAT is used in clinical laboratories ${ }^{46}$ and assays have been developed to detect a number of pathogens including Influenza $\mathrm{A},{ }^{47}$ Escherichia coli, ${ }^{48}$ Helicobacter pylori, ${ }^{49}$ Haemophilus influen$z a e^{50}$ Neisseria meningitidis, ${ }^{51}$ and Streptococcus pneumoniae, ${ }^{52}$ and therefore, it could readily be adopted by existing personnel with minimal training required to implement it. Although the current analysis time at $\sim 18 \mathrm{~h}$ is much longer than the current LAT that use latex beads (due to the speed at which E. coli cells sediment), the application of centrifugation or filtration can be used to accelerate the visualization. Preliminary results using a different biomarker suggest that centrifugation for as little as $20 \mathrm{~min}$ is sufficient to lead to a visible cell pellet (data not shown). Alternatively, changing variables such as $\mathrm{pH}$, ionic strength, and temperature in the reaction medium could possibly decrease the operation time. ${ }^{5}$

In order to increase the portability of the whole-cell biosensor for field-based application, cells could be freezedried and then reconstituted at the site of testing as was shown in other work. ${ }^{19}$ Alternatively, a nonliving alternative could be used, e.g., "bacterial ghost" technology where the surface of cells remains intact, but the cytoplasmic contents are absent. ${ }^{53}$ This would also prevent the accidental release of genetically modified organisms or naked DNA into the field, which would be an additional advantage.

Currently, the whole-cell LAT technology we describe has been tested with a mock patient sample in the form of fibrinogen-spiked human plasma. Further testing of the technology with clinical samples will aid in investigating any possible unidentified interactions in the complex context of biological samples that may interfere with reliable assay functionality.

\section{METHODS}

Strains, Plasmids, and Cell Culture Conditions. Escherichia coli DH10B (F- mcrA $\Delta$ (mrr-hsdRMS-mcrBC) $\varphi 80$ lacZ $\Delta$ M15 $\Delta$ lacX74 recAl endA1 araD139 $\Delta$ (ara-leu) 7697 galU galK $\lambda-\operatorname{rpsL}(\operatorname{StrR}))$ was used in this study. The pNVgfp ${ }^{27}$ and pNVFIB1-2 plasmids, ${ }^{25}$ which express the anti-GFP nanobody and two variants of an anti-human fibrinogen nanobody surface displayed through a $\beta$-intimin, respectively, are described elsewhere. The pNVgfp02-10 constructs were assembled by standard cloning procedures from the pNVgfp construct by replacing the $\mathrm{T} 7$ promoter of the original plasmid with a BBa_J2310x constitutive promoter and a designed RBS element.

For nanobody expression from pNVgfp and pNVFIB1-2, a single colony of freshly transformed DH10B cells was inoculated into LB medium supplemented with $34 \mu \mathrm{g} / \mathrm{mL}$ chloramphenicol and grown for $2 \mathrm{~h}$ at $37^{\circ} \mathrm{C}$ with shaking at $250 \mathrm{rpm}$. For anti-GFP nanobody expression, cell cultures were induced with $1 \mathrm{mM}$ IPTG and incubated at $30{ }^{\circ} \mathrm{C}$ for $3 \mathrm{~h}$ and $250 \mathrm{rpm}$ shaking. Cells were kept 
overnight at $4{ }^{\circ} \mathrm{C}$, then harvested by centrifugation, washed twice, and resuspended in sterile phosphate buffered saline (PBS) $\mathrm{pH} 7.4$ to a final OD600 of $1\left(\sim 1 \times 10^{9}\right.$ cells $)$. The pNVgfp02-10 library of variants utilizes constitutive promoters, and in this case, cell cultures were not induced with IPTG, but instead were grown for $5 \mathrm{~h}$ at $37^{\circ} \mathrm{C}$ with shaking at $250 \mathrm{rpm}$. For anti-fibrinogen nanobody expression, cell cultures were induced with $1 \mathrm{mM}$ IPTG and incubated overnight at $37{ }^{\circ} \mathrm{C}$ with shaking at $250 \mathrm{rpm}$ before cells were harvested by centrifugation. Cells were washed twice and resuspended in sterile phosphate buffered saline (PBS) $\mathrm{pH} 7.4$ to a final OD600 of $1(\sim 1 \times$ $10^{9}$ cells).

Analysis of Nanobody Surface Expression. A volume of 100 $\mu \mathrm{L}$ of the cell suspension was incubated with 200 pmol of purified GFPmut3b at room temperature for $30 \mathrm{~min}$ (for pNVgfp) or $2 \mu \mathrm{L}$ of $50 \mu \mathrm{g} / \mathrm{mL}$ Myc-Tag (9B11) mouse mAb (Alexa Fluor 488 conjugate, Cell Signaling Technology, Danvers, MA) at $4{ }^{\circ} \mathrm{C}$ in the dark for 30 min (for pNVFIB). Cells were washed twice and resuspended in 100 $\mu \mathrm{L}$ of PBS. Subsequently, $2 \mu \mathrm{L}$ of labeled cells was placed on a microscope slide and examined under a Nikon Eclipse Ti inverted microscope (Nikon Instruments, Japan) with exposure time at $100 \mathrm{~ms}$ using a magnification of $60 \times$ with a Ph1 filter.

Nanobody Quantification. The levels of cell-surface displayed nanobody were determined indirectly by measuring the cell fluorescence of bacterial cell populations labeled with different concentrations of purified GFPmut3b. A volume of $100 \mu \mathrm{L}$ of the cell suspension was incubated with various amounts of purified GFP $(0-40 \mathrm{pmol})$ at room temperature for $30 \mathrm{~min}$. Subsequently, cells were washed twice and resuspended in $100 \mu \mathrm{L}$ of PBS. The labeled cells were diluted 1:100 in PBS and analyzed by flow cytometry (Attune NxT flow cytometer, Thermo Fisher Scientific, USA) with at least 100,000 events. Samples were measured at excitation $488 \mathrm{~nm}$ and emission $530 / 30 \mathrm{~nm}$, and data analysis from three replicates was performed using FlowJo software (vX.0.7, FlowJo, LLC, USA). Recorded events were gated using the ellipse gate function to select for single cells at forward scatter (FSC-H) values $629-23,000$ and side scatter (SSC-H) values $6300-58,000$. These values were chosen for providing a large coverage of the cell population events recorded. The standard deviation of the mean cell fluorescence for each sample was calculated using Prism 7 software tool (GraphPad, USA) using geometric mean fluorescence values from three technical replicates of the flow cytometry measurements.

Biological LAT. A volume of $200 \mu \mathrm{L}$ of the cell suspension was incubated with $20-50 \mu \mathrm{L}$ of various concentrations of target molecules in a clear, round-bottom 96-well plate (Costar, USA). Prepared microplates were statically incubated for $\sim 18 \mathrm{~h}$ at room temperature. Assay results were documented using a photographic camera in one or more of the following photography setups: (i) a microplate was placed on top of a dark surface, (ii) a microplate was placed on top of a blue LED transilluminator without the use of blue light filter, and (iii) a microplate was placed on top of a blue LED transilluminator and the screen for blue light absorption was placed on top of the microplate. As for the results testing with hFib, a microplate was placed on top of a light box and photos were taken at three to six wells per frame using a mobile phone.

Fibrinogen Mock Samples. Human fibrinogen (hFib) (Plasminogen, vWF, and Fibronectin depleted; FIB3) was purchased from Enzyme Research Laboratories and diluted in PBS or in fibrinogendeficient human plasma (Sekisui Diagnostics GmbH, Germany). For the plasma-based assays, fibrinogen stock solutions were made at $\sim 5$ $\mathrm{mg} / \mathrm{mL}$ in plasma and then diluted to $1 \mathrm{mg} / \mathrm{mL}$ in PBS.

Mathematical Model and Computer Simulations. The derivation of the mathematical model is described in Supplementary Note 3. The model was implemented in Matlab software using a third-party script ${ }^{54}$ for system simulations and sensitivity analysis.

\section{ASSOCIATED CONTENT}

Supporting Information

The Supporting Information is available free of charge on the ACS Publications website at DOI: 10.1021/acssensors.8b01163.

Supplementary methods, figures, and description of the mathematical model (PDF)

\section{AUTHOR INFORMATION}

Corresponding Authors

*E-mail: p.freemont@imperial.ac.uk. Telephone: (44) 02075945327.

*E-mail: k.polizzi@imperial.ac.uk. Telephone: (44) 02075942851.

ORCID 우

Luis Ángel Fernández: 0000-0001-5920-0638

Paul S. Freemont: 0000-0002-5658-8486

Karen M. Polizzi: 0000-0001-5435-2667

\section{Author Contributions}

Study design: N.K., K.M.P., and P.S.F. Experimental execution and data analysis: N.K., P.R., and H.-E.L. Mathematical modeling: H.E.L. and G.-B.V.S. Nanobody development: V.S. and L.A.F. Writing manuscript: N.K., P.R., H.-E.L., and K.M.P. in consultation with the other authors. All authors have given approval to the final version of the manuscript.

\section{Notes}

The authors declare no competing financial interest.

\section{ACKNOWLEDGMENTS}

We would like to thank the following funding sources: EPSRC EP/K038648/1 (K.M.P. and P.S.F.); BBSRC CASE Studentship (N.K.); Royal Thai Government Scholarship, ${ }^{6}$ MICIU/ AEI/FEDER, EU BIO2017-89081R (L.A.F.); EPSRC EP/ P009352/1 and EP/M002187/1 (G.-B.V.S.); Imperial College President's PhD Scholarship. ${ }^{18}$

\section{ABBREVIATIONS}

tdGFP, tandem dimeric green fluorescent protein; GFP, green fluorescent protein; hFib, human fibrinogen; IPTG, isopropyl$\beta$-thiogalactoside; LAT, latex agglutination test; $\mathrm{Nb}$, nanobody; PBS, phosphate buffered saline.

\section{REFERENCES}

(1) Daar, A. S.; Thorsteinsdottir, H.; Martin, D. K.; Smith, A. C.; Nast, S.; Singer, P. A. Top ten biotechnologies for improving health in developing countries. Nat. Genet. 2002, 32 (2), 229-32.

(2) Giljohann, D. A.; Mirkin, C. A. Drivers of biodiagnostic development. Nature 2009, 462 (7272), 461-4.

(3) Urdea, M.; Penny, L. A.; Olmsted, S. S.; Giovanni, M. Y.; Kaspar, P.; Shepherd, A.; Wilson, P.; Dahl, C. A.; Buchsbaum, S.; et al. Requirements for high impact diagnostics in the developing world. Nature 2006, 444 (Suppl1), 73-9.

(4) Borrebaeck, C. A. Antibodies in diagnostics - from immunoassays to protein chips. Immunology today 2000, 21 (8), 379-82.

(5) Ortega-Vinuesa, J. L.; Bastos-Gonzalez, D. A review of factors affecting the performances of latex agglutination tests. J. Biomater. Sci., Polym. Ed. 2001, 12 (4), 379-408.

(6) Price, C. P. Agglutination techniques for detecting antigenantibody reactions. In eLS; John Wiley \& Sons, Ltd, 2001 (https:// doi.org/10.1038/npg.els.0001114). 
(7) van der Meer, J. R.; Belkin, S. Where microbiology meets microengineering: design and applications of reporter bacteria. Nat. Rev. Microbiol. 2010, 8 (7), 511-22.

(8) Goers, L.; Ainsworth, C.; Goey, C. H.; Kontoravdi, C.; Freemont, P. S.; Polizzi, K. M. Whole-cell Escherichia coli lactate biosensor for monitoring mammalian cell cultures during biopharmaceutical production. Biotechnol. Bioeng. 2017, 114 (6), 1290-1300.

(9) Horswell, J.; Dickson, S. Use of biosensors to screen urine samples for potentially toxic chemicals. J. Anal. Toxicol. 2003, 27 (6), 372-6.

(10) Roda, A.; Pasini, P.; Mirasoli, M.; Guardigli, M.; Russo, C.; Musiani, M.; Baraldini, M. Sensitive determination of urinary mercury(II) by a bioluminescent transgenic bacteria-based biosensor. Anal. Lett. 2001, 34 (1), 29-41.

(11) Turner, K.; Xu, S.; Pasini, P.; Deo, S.; Bachas, L.; Daunert, S. Hydroxylated polychlorinated biphenyl detection based on a genetically engineered bioluminescent whole-cell sensing system. Anal. Chem. 2007, 79 (15), 5740-5.

(12) Courbet, A.; Endy, D.; Renard, E.; Molina, F.; Bonnet, J. Detection of pathological biomarkers in human clinical samples via amplifying genetic switches and logic gates. Sci. Transl. Med. 2015, 7 (289), $289 \mathrm{ra} 83$.

(13) Goers, L.; Kylilis, N.; Tomazou, M.; Wen, K.; Freemont, P.; Polizzi, K. In Microbial Synthetic Biology, 1st ed.; Colin, H., Anil, W., Eds.; Elsevier, 2013; Vol. 40, pp 119-156.

(14) Assicot, M.; Gendrel, D.; Carsin, H.; Raymond, J.; Guilbaud, J.; Bohuon, C. High serum procalcitonin concentrations in patients with sepsis and infection. Lancet 1993, 341 (8844), 515-8.

(15) Haverkate, F.; Thompson, S. G.; Pyke, S. D.; Gallimore, J. R.; Pepys, M. B. Production of C-reactive protein and risk of coronary events in stable and unstable angina. European Concerted Action on Thrombosis and Disabilities Angina Pectoris Study Group. Lancet 1997, 349 (9050), 462-6.

(16) Ohman, E. M.; Armstrong, P. W.; Christenson, R. H.; Granger, C. B.; Katus, H. A.; Hamm, C. W.; O’Hanesian, M. A.; Wagner, G. S.; Kleiman, N. S.; et al. Cardiac troponin T levels for risk stratification in acute myocardial ischemia. GUSTO IIA Investigators. N. Engl. J. Med. 1996, 335 (18), 1333-41.

(17) Thompson, I. M.; Pauler, D. K.; Goodman, P. J.; Tangen, C. M.; Lucia, M. S.; Parnes, H. L.; Minasian, L. M.; Ford, L. G.; Lippman, S. M.; et al. Prevalence of prostate cancer among men with a prostate-specific antigen level $<$ or $=4.0 \mathrm{ng}$ per milliliter. N. Engl. J. Med. 2004, 350 (22), 2239-46.

(18) Looger, L. L.; Dwyer, M. A.; Smith, J. J.; Hellinga, H. W. Computational design of receptor and sensor proteins with novel functions. Nature 2003, 423 (6936), 185-90.

(19) Webb, A. J.; Kelwick, R.; Doenhoff, M. J.; Kylilis, N.; MacDonald, J. T.; Wen, K. Y.; McKeown, C.; Baldwin, G.; Ellis, T.; et al. A protease-based biosensor for the detection of schistosome cercariae. Sci. Rep. 2016, 6, 24725.

(20) Daugherty, P. S.; Chen, G.; Olsen, M. J.; Iverson, B. L.; Georgiou, G. Antibody affinity maturation using bacterial surface display. Protein Eng., Des. Sel. 1998, 11 (9), 825-32.

(21) Francisco, J. A.; Campbell, R.; Iverson, B. L.; Georgiou, G. Production and fluorescence-activated cell sorting of Escherichia coli expressing a functional antibody fragment on the external surface. Proc. Natl. Acad. Sci. U. S. A. 1993, 90 (22), 10444-8.

(22) Fuchs, P.; Breitling, F.; Dubel, S.; Seehaus, T.; Little, M. Targeting recombinant antibodies to the surface of Escherichia coli: fusion to a peptidoglycan associated lipoprotein. Bio/Technology 1991, 9 (12), 1369-72.

(23) Muyldermans, S. Nanobodies: natural single-domain antibodies. Annu. Rev. Biochem. 2013, 82, 775-97.

(24) Salema, V.; Fernandez, L. A. Escherichia coli surface display for the selection of nanobodies. Microb. Biotechnol. 2017, 10 (6), 146884.

(25) Salema, V.; Lopez-Guajardo, A.; Gutierrez, C.; Mencia, M.; Fernandez, L. A. Characterization of nanobodies binding human fibrinogen selected by E. coli display. J. Biotechnol. 2016, 234, 58-65.
(26) Salema, V.; Manas, C.; Cerdan, L.; Pinero-Lambea, C.; Marin, E.; Roovers, R. C.; van Bergen En Henegouwen, P. M.; Fernandez, L. A. High affinity nanobodies against human epidermal growth factor receptor selected on cells by E. coli display. $m A$ abs 2016, 8 (7), 12861301.

(27) Salema, V.; Marin, E.; Martinez-Arteaga, R.; Ruano-Gallego, D.; Fraile, S.; Margolles, Y.; Teira, X.; Gutierrez, C.; Bodelon, G.; Fernandez, L. A. Selection of single domain antibodies from immune libraries displayed on the surface of $\mathrm{E}$. coli cells with two betadomains of opposite topologies. PLoS One 2013, 8 (9), No. e75126.

(28) Cavallari, M. Rapid and Direct VHH and Target Identification by Staphylococcal Surface Display Libraries. Int. J. Mol. Sci. 2017, 18 (7), No. 1507.

(29) Fleetwood, F.; Devoogdt, N.; Pellis, M.; Wernery, U.; Muyldermans, S.; Stahl, S.; Lofblom, J. Surface display of a singledomain antibody library on Gram-positive bacteria. Cell. Mol. Life Sci. 2013, 70 (6), 1081-93.

(30) Willis, A.; Jung, E. J.; Wakefield, T.; Chen, X. Mutant p53 exerts a dominant negative effect by preventing wild-type p 53 from binding to the promoter of its target genes. Oncogene 2004, 23 (13), 2330-8.

(31) Lowe, G. D.; Rumley, A.; Mackie, I. J. Plasma fibrinogen. Ann. Clin. Biochem. 2004, 41 (6), 430-40.

(32) NICE In 1.1 Presentation with lower respiratory track infection; National Institute for Health and Care Excellence, 2014.

(33) Dolgosheina, E. B.; Karulin, A.; Bobylev, A. V. A kinetic model of the agglutination process. Math. Biosci. 1992, 109 (1), 1-10.

(34) Registry of Standard Biological_Parts, 2016 http://parts.igem. org/Promoters/Catalog/Anderson (accessed 2nd October 2018).

(35) Salis, H. M.; Mirsky, E. A.; Voigt, C. A. Automated Design of Synthetic Ribosome Binding Sites to Precisely Control Protein Expression. Nat. Biotechnol. 2009, 27 (10), 946.

(36) Mackie, I. J.; Kitchen, S.; Machin, S. J.; Lowe, G. D. O. Guidelines on fibrinogen assays. Br. J. Haematol. 2003, 121 (3), 39650.

(37) Daringer, N. M.; Dudek, R. M.; Schwarz, K. A.; Leonard, J. N. Modular extracellular sensor architecture for engineering mammalian cell-based devices. ACS Synth. Biol. 2014, 3 (12), 892-902.

(38) Ostrov, N.; Jimenez, M.; Billerbeck, S.; Brisbois, J.; Matragrano, J.; Ager, A.; Cornish, V. W. A modular yeast biosensor for low-cost point-of-care pathogen detection. Science advances 2017, 3 (6), No. e1603221.

(39) Harmsen, M. M.; De Haard, H. J. Properties, production, and applications of camelid single-domain antibody fragments. Appl. Microbiol. Biotechnol. 2007, 77 (1), 13-22.

(40) Tanha, J.; Dubuc, G.; Hirama, T.; Narang, S. A.; MacKenzie, C. R. Selection by phage display of llama conventional $\mathrm{V}(\mathrm{H})$ fragments with heavy chain antibody $\mathrm{V}(\mathrm{H}) \mathrm{H}$ properties. J. Immunol. Methods 2002, 263 (1-2), 97-109.

(41) Verheesen, P.; Roussis, A.; de Haard, H. J.; Groot, A. J.; Stam, J. C.; den Dunnen, J. T.; Frants, R. R.; Verkleij, A. J.; Theo Verrips, C.; van der Maarel, S. M. Reliable and controllable antibody fragment selections from Camelid non-immune libraries for target validation. Biochim. Biophys. Acta, Proteins Proteomics 2006, 1764 (8), 1307-19.

(42) van der Linden, R.; de Geus, B.; Stok, W.; Bos, W.; van Wassenaar, D.; Verrips, T.; Frenken, L. Induction of immune responses and molecular cloning of the heavy chain antibody repertoire of Lama glama. J. Immunol. Methods 2000, 240 (1-2), 185-195.

(43) Gatgan, P. E.; Sheehan, C.; Williams, L.; Ploplis, V.; Hearty, U.; Lowry, C. A rapid latex agglutination assay for the determination of plasma fibrinogen. Blood Coagulation Fibrinolysis 1990, 1 (5), 465-8.

(44) Chen, Q.; Hua, X.; Fu, W.; Liu, D.; Chen, M.; Cai, G. Quantitative determination of fibrinogen of patients with coronary heart diseases through piezoelectric agglutination sensor. Sensors 2010, 10 (3), 2107-18.

(45) Campuzano, S.; Salema, V.; Moreno-Guzman, M.; Gamella, M.; Yanez-Sedeno, P.; Fernandez, L. A.; Pingarron, J. M. Disposable amperometric magnetoimmunosensors using nanobodies as biore- 
cognition element. Determination of fibrinogen in plasma. Biosens. Bioelectron. 2014, 52, 255-60.

(46) Mikkelsen, S. R.; Carton, E. In Bioanalytical Chemistry, 2nd ed.; Wiley: Germany, 2016, DOI: 10.1002/0471623628.ch6.

(47) Eisfeld, A. J.; Neumann, G.; Kawaoka, Y. Influenza A virus isolation, culture and identification. Nat. Protoc. 2014, 9, 2663-81.

(48) Rocha, L. B.; Santos, A. R. R.; Munhoz, D. D.; Cardoso, L. T. A.; Luz, D. E.; Andrade, F. B.; Horton, D. S. P. Q.; Elias, W. P.; Piazza, R. M. F. Development of a Rapid Agglutination Latex Test for Diagnosis of Enteropathogenic and Enterohemorrhagic Escherichia coli Infection in Developing World: Defining the Biomarker, Antibody and Method. PLoS Neglected Trop. Dis. 2014, 8 (9), No. e3150.

(49) Tsutsumi, K.; Kusano, C.; Suzuki, S.; Gotoda, T.; Murakami, K. Diagnostic Accuracy of Latex Agglutination Turbidimetric Immunoassay in Screening Adolescents for Helicobacter pylori Infection in Japan. Digestion 2018, 98 (2), 75-80.

(50) Heikkilä, R.; Takala, A.; Käyhty, H.; Leinonen, M. Latex agglutination test for screening of Haemophilus influenzae type $\mathrm{b}$ carriers. J. Clin. Microbiol. 1987, 25 (6), 1131-3.

(51) Leinonen, M.; Herva, E. The latex agglutination test for the diagnosis of meningococcal and Haemophilus influenzae meningitis. Scand. J. Infect. Dis. 1977, 9 (3), 187-91.

(52) Smith, S. K.; Washington, J. A. Evaluation of the Pneumoslide latex agglutination test for identification of Streptococcus pneumoniae. J. Clin. Microbiol. 1984, 20 (3), 592-3.

(53) Langemann, T.; Koller, V. J.; Muhammad, A.; Kudela, P.; Mayr, U. B.; Lubitz, W. The bacterial ghost platform system. Bioengineered Bugs 2010, 1 (5), 326-36.

(54) Garcia Molla, V. M.; Gomez Padilla, R. Sensitivity analysis for ODEs and DAEs. 2002, https://uk.mathworks.com/matlabcentral/ fileexchange/1480-sensitivity-analysis-for-odes-and-daes. Accessed June 2, 2018 